\title{
Novel Characterization of Deformation Mechanisms in a Ni-base Superalloy Using HAADF Imaging and Atomic Ordering Analysis
}

\author{
Donald McAllister ${ }^{1}$, Duchao Lv ${ }^{1}$, Hallee Deutchman ${ }^{2}$, Benjamin Peterson ${ }^{2}$, Yunzhi Wang ${ }^{1}$, and Michael \\ J. Mills ${ }^{1}$ \\ 1. The Ohio State University, Department of Materials Science and Engineering, Columbus, Ohio, USA \\ 2. Honeywell Aerospace, Phoenix, Arizona, USA
}

Ni-base superalloy 718 is a prominent material used for disk components in modern turbine engines. Though there is an extensive database of processing/property relationships that has been developed over its decades of use, there is little consensus on the deformation mechanisms present in 718 over its usable temperature range [1]. The asymmetry of the $\mathrm{D}_{22} \gamma^{\prime \prime}$ strengthening phase has been shown to cause numerous deformation modes in literature, including microtwinning, stacking fault formation, and the cooperative motion of like-signed $1 / 2\langle 110\rangle$ dislocations [1,2] in overaged samples. In this work, the post-deformation dislocation structure are analyzed in order to inform the creation of mechanisticallyaccurate yield strength models in commercially-relevant materials.

Initial bright field and dark field scanning transmission electron microscopy (STEM) results indicated that simple APB shearing by single $1 / 2<110>$ dislocation is not an operative mechanism [3]. In fact, the strain contrast analysis indicates some form of $\langle 112\rangle$ type dislocation is operative in alloy 718 . Atomic resolution high angle annular dark field (HAADF) STEM was utilized to investigate the local strains and shear structure. The interactions between the $\gamma^{\prime \prime}$ phase and the shearing dislocations were evident, and the local fault structure in the $\gamma^{\prime \prime}$ was characterized through the intensity of the Nb-rich columns in " $Z$ " contrast HAADF STEM. Combined with knowledge of the energy landscape produced by ab-initio calculations of the generalized stacking fault, the shear history of the particle could be deduced [3].

The unique benefit of HAADF STEM is exploited in characterizing the shearing of composite morphologies of the $\gamma^{\prime \prime}$ phase. The $\gamma^{\prime \prime}$ and the $\gamma^{\prime}$ phase commonly form composite particles with the $(001)_{\gamma^{\prime \prime}}$ parallel to the three $\{100\}_{\gamma^{\prime}}$ planes [4]. The phase boundary forms as an atomically-planar interface which is visible in HAADF STEM as a low intensity plane. The low intensity is likely due to the segregation of aluminum to the phase boundary [5]. This low intensity plane acts as an inert marker that can be tracked to determine the shear history of the particle along a single plane. This has been used to effectively show the complex deformation mechanisms present in alloy 718 . The exact dislocations that were necessary to shear particles into the visible stacking sequence were determined for both single layer stacking faults as well as twins.

Many stacking fault sequences predicted through generalized stacking fault analysis and phase field modeling were confirmed through atomic analysis of the $\gamma^{\prime \prime}$ fault structure. The formation of intrinsic stacking faults was found in the $\gamma^{\prime \prime}$ at all temperatures. The faulting was isolated to the $\gamma^{\prime \prime}$ at $22^{\circ} \mathrm{C}$, but it extended into the matrix during deformation at $427^{\circ} \mathrm{C}$. Microtwins formed during deformation at $427^{\circ} \mathrm{C}$, but they were limited to the $\gamma^{\prime \prime}$ phase. Deformation at $649^{\circ} \mathrm{C}$ allowed for extensive microtwinning, and the microtwins that formed were not the result of multiple like-signed partial dislocations. Additionally, multiple unlike $1 / 2<110\rangle$ dislocations were active on a single shear planes at all temperatures. 
High resolution energy dispersive spectroscopy (EDS) was used to understand the formation of twins. It was shown that there was no discernable segregation to the twins within the matrix. Additionally, the location of the microtwins and their interactions with the $\gamma^{\prime}$ and $\gamma^{\prime \prime}$ phases were identified through a combination of angular dark field STEM and EDS.

\section{References:}

[1] Sundararaman, M. et al.. Acta Metall. 36, 847-864 (1988).

[2] McAllister, D. et al. Scr. Mater. 115, 108-112 (2016).

[3] Phillips, P. J. et al. Appl. Phys. Lett. 100, 211913 (2012).

[4] Geng, W. et al. Phys. Rev. B 76, 224102 (2007).
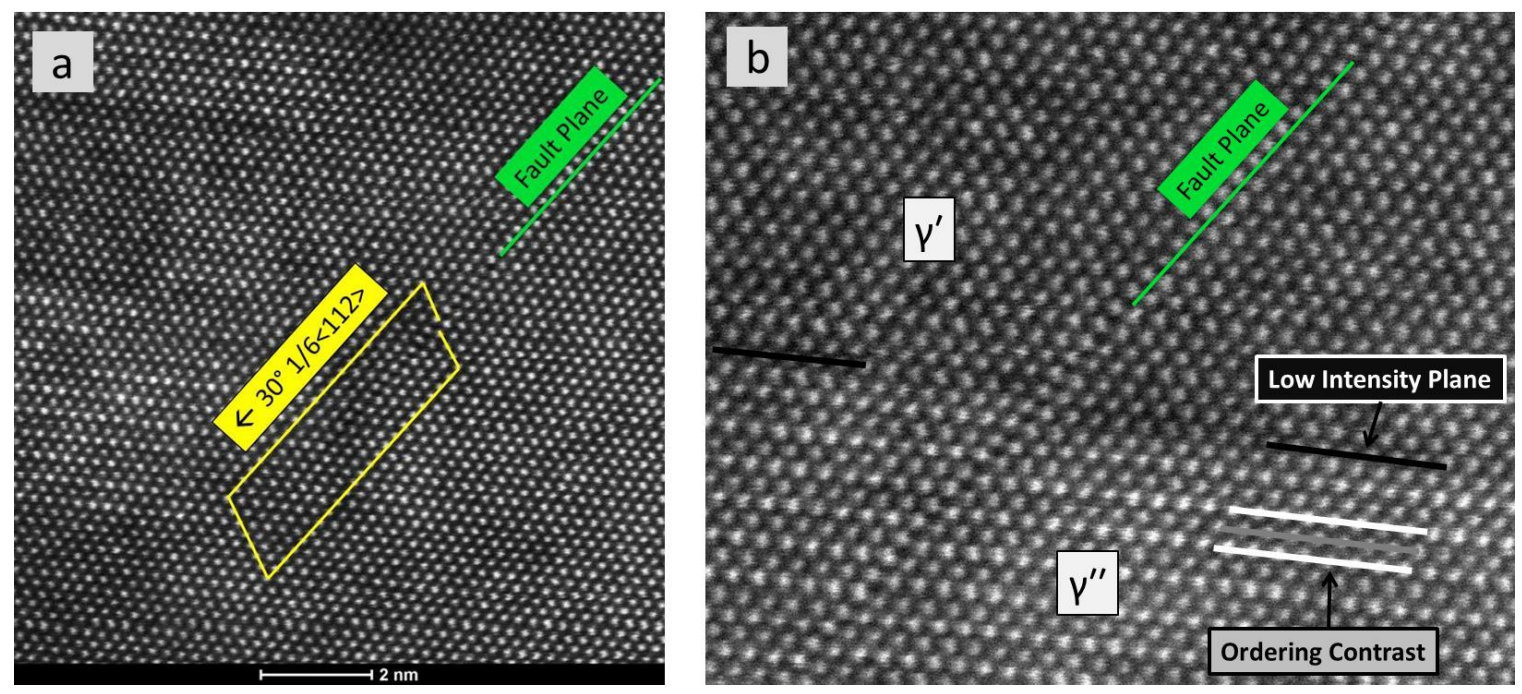

Figure 1. Atomic resolution analysis of the leading partial (a) and ordering sequence (b) of a single fault through the $\gamma^{\prime \prime}$ phase after deformation at $427^{\circ} \mathrm{C}$.
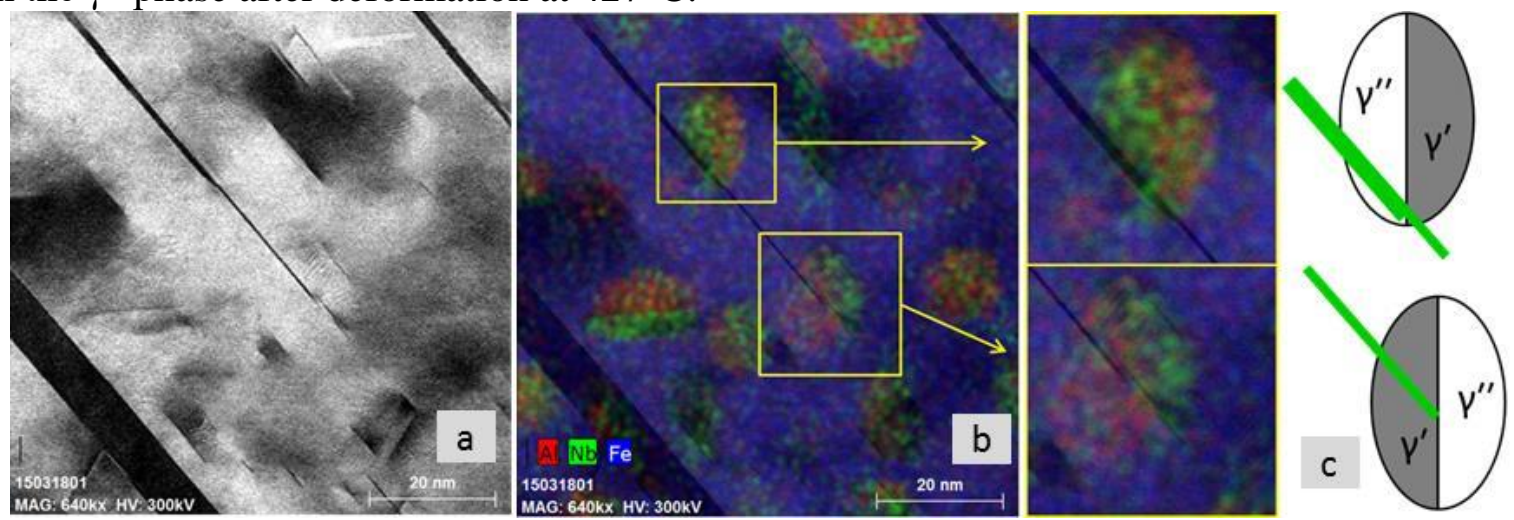

Figure 2. High Resolution ADF STEM (a) showing the positions of twins overlaid with chemical information from EDS (b) and schematics showing microtwin interaction with composite particles (c). 